\title{
Annihilation Radii for Dislocations Intercepting a Free Surface with Application to Heteroepitaxial Thin Film Growth
}

\author{
M. Chang, S.K. Mathis*, G.E. Beltz, C.M. Landis \\ Department of Mechanical and Environmental Engineering, *Department of Materials \\ University of California, Santa Barbara, CA 93 106-5070
}

\begin{abstract}
One critical issue in heteroepitaxial, lattice mismatched growth is the inevitable appearance of threading dislocations which reside in the relaxing film and degrade its semiconducting properties. It has been shown in previous work that threading dislocations interact with each other through a series of annihilation and fusion reactions to decrease their density as the film thickness increases and follow a $1 / h$ decay, where $h$ is the film thickness. A characteristic reaction radius is associated with these interactions. In previous simulations, the reaction radius was taken to be a constant value estimated using a simple approximation based on infinite, parallel dislocation lines. Here, a continuum-based elasticity approach is taken to more accurately quantify the reaction radius by comparing the Peach-Koehler force of one dislocation acting on another at a free surface with the lattice resistance to dislocation motion. The presence of the free surface gives rise to a moderate reduction of the interaction force. Results are compared with preliminary experimental data for $\mathrm{GaAs}$ films grown on $\mathrm{InP}$.
\end{abstract}

\section{INTRODUCTION AND BACKGROUND}

During heteroepitaxial growth of thin-films for electronic devices, misfit dislocations (MD) inevitably nucleate due to lattice mismatch stresses. Upon reaching a critical thickness, $h$, the film "relaxes" as misfit dislocations, and associated threading dislocations, appear and multiply. Misfit dislocations are equilibrium defects - i.e. they are necessary to relieve mismatch strain, while threading dislocations are non-equilibrium line defects that link a given MD to the surface. Threading dislocation densities for films with large mismatch (in excess of $-2 \%$ ) can be on the order of $10^{0}-10^{11} \mathrm{~cm}^{-2}$ and as pointed out by Beltz et al. [1], their population is largely reducible while retaining the necessary MD density to sustain a relaxed film. A wealth of experimental work has been performed in an attempt to reduce TD densities. However, relatively few theoretical efforts have been carried out to understand TD density reduction mechanism.

There have been several experimental research efforts to characterize TD density reduction with film thickness for several material systems. Tachikawa and Yamaguchi [2] observed a 1/h dependence of TD density in GaAs films on Si substrate, where h is the film thickness. TD reduction has also been reported for InAs/GaAs, GaAs/Ge/Si, GaAs/InP, and InAs/InP by Sheldon et al. [3], showing similar $1 / h$ dependence for all 4 material systems. They found TD density to be inversely proportional to film thickness for initial TD densities of $10^{8}-10^{9} \mathrm{~cm}^{-2}$. Additionally, the reduction behavior was found to be similar for all of the material systems, indicating that the fundamental mechanics of dislocation reduction are the same regardless of material system. Tachikawa and Yamaguchi also found dislocation density to be exponentially proportional to film thickness for initial TD densities of less than $10^{7} \mathrm{~cm}^{-2}$. Mathis et al. [4] have recently shown TD density data for GaAs grown on InP displaying the $1 / h$ behavior, as shown in Figure 1.

In the recent theoretical work by Beltz et al., a computer simulation was developed to study TD density reduction [1]. TDs are allowed to "react" with one another to reduce their overall density, giving results consistent with the $1 / h$ density dependence and ultimate saturation predicted by earlier models [5] and observed experimentally by such groups as Tachikawa and Yamaguchi. A primary tenet in the theoretical models to date is that TDs within a reaction distance of each other spontaneously interact. Given the correct Burgers vector combination, annihilation $\left(\boldsymbol{b}_{1}+\boldsymbol{b}_{2}=0\right)$ or fusion $\left(\boldsymbol{b}_{1}+\boldsymbol{b}_{2}=\boldsymbol{b}_{3}\right)$ of the TDs could occur, or the dislocations would simply continue following a trajectory to the surface, unimpeded or at most repelled by each other. 
In conjunction with the computer simulation, an analytical approach was also taken to further understand TD reduction. Romanov et al. [6,7] present an analytical model for TD reduction based on the principal of chemical kinetics. They derived and solved a system of nonlinear first order differential equations for the 24 types of TDs for FCC materials. Their numerical results recover the $1 / h$ dependence as well as the saturation behavior that has been observed both experimentally and in simulations.

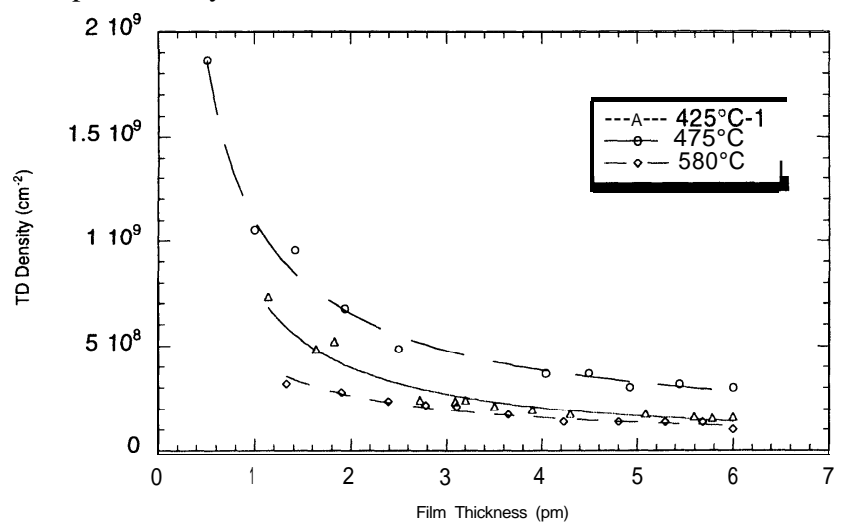

Figure 1. Threading dislocation density data for GaAs grown on InP via MBE.

\section{SIMPLE REACTION RADIUS ESTIMATION}

In the aforementioned theoretical work, the most critical physical parameters were left as unknowns against which the primary results were normalized. The reaction radius for annihilation and fusion reactions were assumed to be equivalent and were estimated by comparing the force acting between two dislocations, also known as the Poech-Koehler force, with the force opposing dislocation movement due to the lattice, also known as the Peierls stress. Since TDs are substantially of screw character, only the conditions for screw dislocations are considered here for simplicity. For the simple case of two parallel screw dislocations, the magnitude of the PK force is:

$$
F^{P K}=\frac{\mu b^{2}}{2 \pi r}
$$

The lattice friction force per unit length of dislocation due to the Peierls stress, $\sigma_{\mathrm{p}}$, is given as:

$$
F_{p}=\sigma_{p} b
$$

Dislocation motion will occur when these two forces are equivalent. Thus, the reaction radius, $\mathrm{r}_{\mathrm{A}}$, is approximated as:

$$
r_{A}=\frac{\mu b}{2 \pi \sigma_{p}}
$$

Based on the parameters of typical semiconductor materials, $\mathrm{r}_{\mathrm{A}}$ was set at $5000 \AA$ [ [1]. Figure 2 shows results by Mathis $e t$ al. [4] of the reaction radius from the GaAs on InP system previously mentioned, with the reaction radius varying from $\sim 100-1200 \mathrm{~A}$, agreeing fairly well with the predicted range. Since dislocation mobility increases with temperature, it is physically realistic for the annihilation radius to increase with temperature as shown in Figure 2. Peierls stresses for a few representative materials are listed in Table 1. 


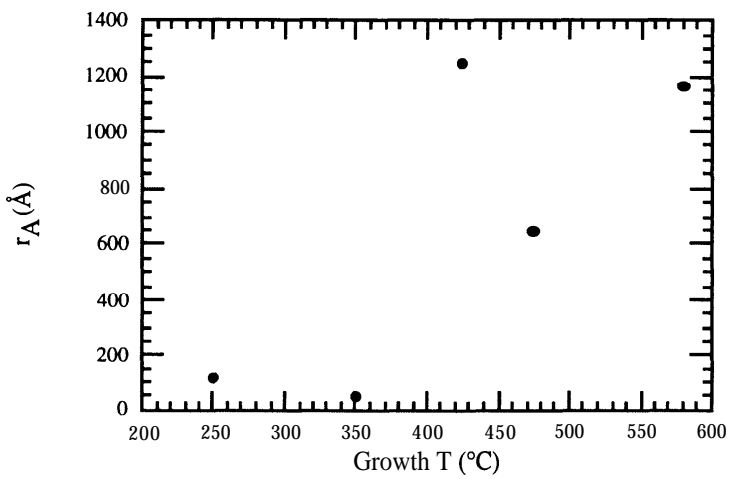

Figure 2. Experimental data for GaAs on InP system showing annihilation radii at various growth temperatures.

Table 1. Peierls stress and shear modulus for various materials.

\begin{tabular}{|c|c|c|c|c|}
\hline M aterial & Peierls Stress (MPa) & $\sigma / \mu(\times 10-3)$ & $\mu(\mathbf{G P a})$ & $\mathbf{T}(\mathbf{K})$ \\
\hline Silicon [6] & 110 (expt) & 2.62 & 42 & 873 \\
\hline $\mathrm{ZrO}$ [7] & $100-150$ (expt) & $1.25-1.88$ & 80 & 293 \\
\hline $\mathrm{GaAs}[8]$ & $35-65$ & $0.73-1.35$ & 48 & 623 \\
\hline $\mathrm{MgO}[9]$ & $60-170$ (expt) & $0.69-1.95$ & 87 & $373-423$ \\
\hline
\end{tabular}

\section{ELASTIC STRESS FIELD FOR A GENERAL DISLOCATION PERPENDICULAR TO A SURFACE}

To further enhance the simulation results, we wish to estimate the reaction $\operatorname{radii}, \mathrm{r}_{\mathrm{A}}$ and $\mathrm{r}_{\mathrm{F}}$, using a more rigorous approach from the mechanics of defects. Comparing the elastic force between two dislocations at a free surface with the friction force from the Peierls stress yields a value for the reaction radius. In addition, we get a feel for the range of values for reaction radii for various material classes with an ultimate focus on semiconductor materials.

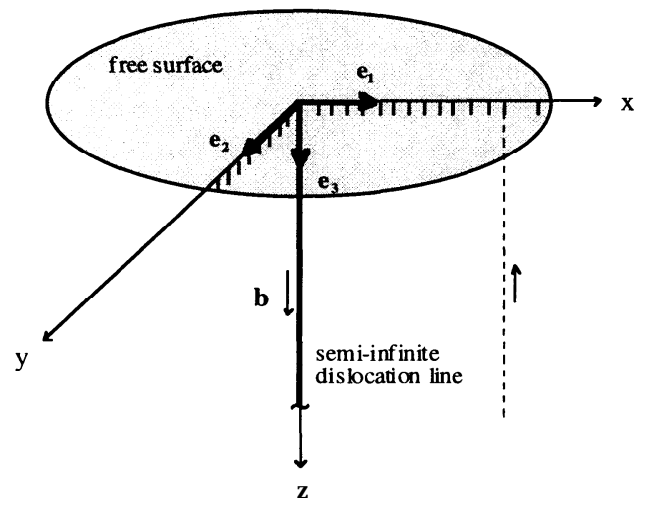

Figure 3. Straight dislocation intersecting a free surface. 
A number of investigators have considered the problem of the stress field due to a straight dislocation intercepting a free surface (see Figure 3). The first, and best known work in this area is that due to Yoffe [10]. Thorough reviews of the methodology used for such solutions are provided by Bacon and Groves [ 11], Eshelby [ 12], Lothe [13], and Yu. Belov [ 14]. Unfortunately, the literature in this problem is infamous for the presence of a moderate number of misprints, especially in the equations for the dislocation stress field. As pointed out by Shaibani and Hazzledine [ 15], the approach of Yoffe lends itself well to concise expressions for the stress fields but misprints exist in that original work. They use Yoffe's method to reconstruct the fields; however, as mentioned by the same authors in a later paper [16], at least one misprint appears in their results. Rather than directly adopting the stress fields provided in the literature, we directly verify them. We derive the stress field due to a screw dislocation perpendicular to a free surface. Components of an edge dislocation perpendicular to a surface have also been derived, but will not be shown here.

Consider the coordinate system shown in Figure 3, with a single right-handed screw dislocation collinear with the $\mathrm{z}$ axis and the half-space occupying $\mathrm{z}>0$. Unit vectors along the three axes are denoted $\mathbf{e}_{i}$, with the usual Einstein index convention. Our definition of "righthanded" is consistent with the usage of Hirth and Lothe [17], in that $\mathbf{e}_{\mathbf{3}} \cdot \mathbf{b}=b$, with the "positive" line sense of the dislocation in the positive $\mathrm{z}$ direction. The stress solution for the infinite screw dislocation,

$$
\sigma_{\theta z}=\frac{\mu b}{2 \pi r}
$$

satisfies all of the boundary conditions for this problem except the traction free condition at $\mathrm{z}=0$; i.e., we require $\sigma_{\theta z}=0$ for $\mathrm{z}=0$. One could correct the solution in Equation (4) by integrating a point force $K\left(\mathbf{r}, \mathbf{r}^{\prime}\right)$ over the entire surface, weighted by the excess stress $\sigma_{\theta z}$ given by Equation (4), thereby nullifying the total traction on the surface. In polar coordinates, $K\left(\mathbf{r}, \mathbf{r}^{\prime}\right)$ gives the stress $\sigma_{\theta \mathrm{z}}$ at position $\mathrm{r}$ due to a point force of unit magnitude applied parallel to the surface and tangential to a circle of radius $r$ ' about the origin and can be constructed from the concentrated tangential force solution found in Johnson [18]. The total stress field is thus given by

$$
\begin{gathered}
\sigma_{\theta z}=\frac{\mu b}{2 \pi r}+\int_{0}^{2 \pi} \int_{0}^{\infty} \frac{\mu b}{2 \pi r^{\prime}} K\left(\boldsymbol{r}, \boldsymbol{r}^{\prime}\right) r^{\prime} d r^{\prime} d \theta^{\prime}=\frac{\mu b z}{2 \pi r \sqrt{r^{2}+z^{2}}} \\
\sigma_{r \theta}=-\frac{\mu b r^{2}}{2 \pi \sqrt{r^{2}+z^{2}}\left(z+\sqrt{r^{2}+z^{2}}\right)^{2}}
\end{gathered}
$$

These stresses agree with those for a screw dislocation in an infinite solid in the limit as $\mathrm{z} \rightarrow \infty$, as well as give a traction-free condition $\sigma_{\mathrm{i} 3}=0$ for $\mathrm{z}=0$. Moreover, they agree with the result of Hazzledine [12], except that care must be taken with the coordinate system, since their screw dislocation occupies the axis $\mathrm{z}<0$. Converting Equation (5) gives the following stress field in Cartesian coordinates:

$$
\begin{array}{ll}
\sigma_{11}=\frac{\mu b}{2 \pi} \frac{2 x y}{R(z+R)^{2}} & \sigma_{12}=\frac{\mu b}{2 \pi} \frac{\left(y^{2}-x^{2}\right)}{R(z+R)^{2}} \\
\sigma_{22}=-\mu b-\frac{2 x y}{2 \pi} \frac{\sigma_{23}}{R(z+R)^{2}} & =\frac{\mu b}{2 \pi} \frac{x z}{R r^{2}} \\
\sigma_{33}=0 & \sigma_{13}=-\frac{\mu b}{2 \pi} \frac{y z}{R r^{2}}
\end{array}
$$


where $\mathrm{r}=\sqrt{\left(x^{2}+y^{2}\right)}$ and $\mathrm{R}=\sqrt{\left(x^{2}+y^{2}+z^{2}\right)}$. As a final check, Equations (6) satisfy the field equations of elasticity for an isotropic material:

$$
\begin{aligned}
& \sigma_{i j, j}=0 \\
& \varepsilon_{i j}=\frac{1}{2 \mu}\left(\sigma_{i j}-\frac{v}{1+v} \sigma_{k k} \delta_{i j}\right) \\
& \varepsilon_{i j, k l}+\varepsilon_{k l, i j}-\varepsilon_{i k, j l}-\varepsilon_{j l, i k}=0
\end{aligned}
$$

The stress field for an edge dislocation intercepting a surface is considerably more difficult to obtain due to the lack of rotational symmetry about the $\mathrm{z}$ axis. Combining the solutions for the screw and edge dislocation gives the stress field for a mixed dislocation impinging a free surface, which we will present in future work.

\section{FREE SURFACE EFFECTS}

The PK force is given by $-\varepsilon_{i j k} s_{i} \sigma_{i l} b_{1}$ where $s_{i}$ are the components of the line sense of the dislocation in Einstein notation. Ev̄alüation gives:

$$
\begin{aligned}
& F_{1}^{P K}=-\frac{\mu b^{2}}{2 \pi} \frac{x z}{r^{2} \sqrt{r^{2}+z^{2}}} \\
& F_{2}^{P K}=-\frac{\mu b^{2}}{2 \pi} \frac{y z}{r^{2} \sqrt{r^{2}+z^{2}}}
\end{aligned}
$$

or expressed as a radial component,

$$
F_{r}^{P K}=\frac{\mu b^{2} z}{2 \pi r \sqrt{r^{2}+z^{2}}}
$$

i.e., a purely attractive force of magnitude $\frac{\mu b^{2} z}{2 \pi r \sqrt{r^{2}+z^{2}}}$. This force is independent of the relative angular position of the dislocations.

Equating the PK force with the Peierls force and solving for $r_{A}$ gives:

$$
\frac{r_{A}}{r_{A_{\infty}}}=\frac{\sqrt{2} \pi \sigma_{p} \bar{z}}{\mu} \sqrt{-1+\sqrt{1+\frac{\mu^{2}}{\pi^{2} \sigma_{p}^{2} \bar{z}^{2}}}}
$$

where $\bar{z}=z / b$. Note that as $z \rightarrow 0$, i.e. the free surface, the attractive force vanishes. Equation (10) is plotted in Figure 4 for various values of $\sigma_{\mathrm{p}} / \mu$.

\section{CONCLUSION}

The annihilation radius for two opposite, parallel screw dislocations is given by $\frac{\mu b}{2 \pi \sigma_{p}}$.

When experimental results on threading dislocation reduction are compared with the theory of Speck et al., the annihilation radius estimated here agrees within one order of magnitude with the value necessary for the theory to match the experiment. The effect of the free surface is to 
diminish the interaction force between the dislocations, as well as the annihilation radius. For reasonable physical parameters, this decay only occurs near of the surface. It should be noted that the existence of any edge component in the reacting dislocations will increase the attractive force between the two dislocations since there is always an attractive force between two edge dislocations, even when $\mathrm{z} \rightarrow 0$. In preliminary work by Beltz et al. [19], the reaction radius for edge dislocations is shown to have a maximum value near the free surface before decaying to steady state.

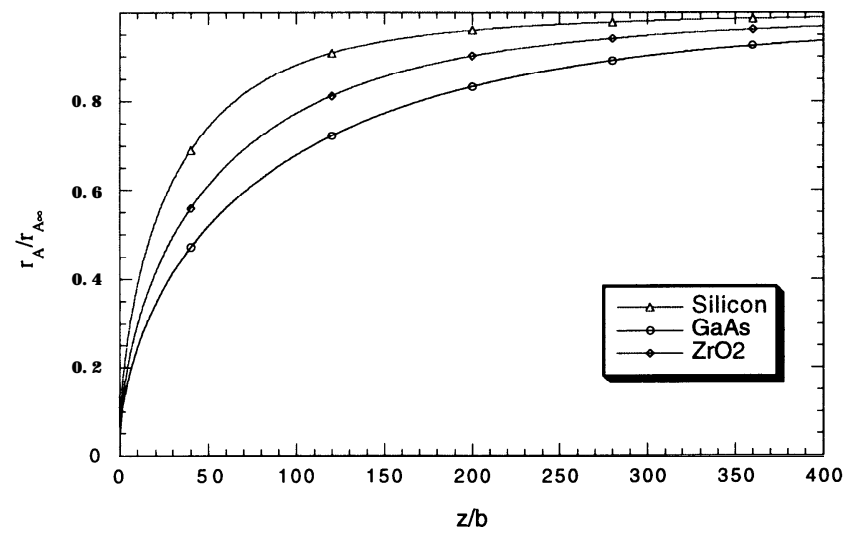

Figure 4. Free surface effect on reaction radius for various materials.

\section{REFERENCES}

[1] G.E. Beltz, M. Chang, J.S. Speck, W. Pompe, A.E. Romanov, Philosophical Magazine A, 76, No. 4, (1997) 807-835.

[2] M. Tachikawa, and M. Yamaguchi, Appl. Phys. Lett. 56, (1990) 484.

[3] P. Sheldon, K.M. Jones, M.M. Al-Jassim, B.G.Jacobi, J. Appl. Phys., 63 (1988) 5609.

[4] SK. Mathis, X.H. Wu, A.E. Romanov, J.S. Speck, submitted to J. Applied Physics.

[5] A.E. Romanov, W. Pompe, G.E. Beltz, J.S. Speck, Appl. Phys. Lett. 69 (1996) 3342.

[6] A.E. Romanov, W. Pompe, G.E. Beltz, J.S. Speck, Phys. Stat. Sol. B, 198 (1996) 599.

[7] A.E. Romanov, W. Pompe, G.E. Beltz, J.S. Speck, Phys. Stat. Sol. B, 199 (1997) 33.

[8] Y .H. Chiao, D.R. Clarke, Acta Metall., 37, No. 1, (1989), No. 1, 203.

[9] Y.H. Chiao, I.W. Chen, Acta Metall., 38, No.6 (1990) 1163.

[10] D. Caillerd, N. Clement, A. Couret, Y. Androussi, A. Lefebvre, G. Vanderschaeve, Inst Phys Conf Ser No.100: Section 5, (1989), 403.

[ 11 ] Singh, R.N., Coble, R.L., J. Appl. Physics, 45, (1974) 981.

[12] E.H. Yoffe, Philosophical Magazine, 6, (1961), 1147.

[13] D.J. Bacon, P.P. Groves, Fundamentals of Aspects of Dislocation Theory, ed. J.A. Simmons, R. deWitt, R. Bullough, National Bureau of Standards Special Publication 3 17, 1, (1969), 35.

[14] J.D. Eshelby, Dislocations in Solids, ed. F.R.N. Nabarro, North-Holland Publishing Co, 1, (1980), 167.

[15] J. Lothe, in: Elastic Strain Fields and Dislocation Mobility, ed. V.L. Indenbom and J. Lothe, Elsevier Science Publishers, (1992), 329.

[16]A. Yu. Belov, in: Elastic Strain Fields and Dislocation Mobility, ed. V.L. Indenbom and J. Lothe, Elsevier Science Publishers, (1992), 391.

[17] S.J. Shaibani, P.M. Hazzeldine, Philosophical Magazine A, 44, No.3.,(1981), 657.

[18] P.M. Hazzeldine, S.J. Shaibani, Strength of Metals and Alloys (ICSMA6), ed. R.C. Gifkins, Proc. of 6th Intl Conf., Pergamon, Oxford, 1 (1983) 45.

[19] L.P. Hirth, J. Lothe, Theory of Dislocations, 2nd ed., Krieger Publishing, (1992).

[20] K.L.Johnson, Contact Mechanics, Cambridge University Press, 1985, 68.

[21] G.E. Beltz, M. Chang, C.M. Landis, work in progress. 